\title{
LE MAROC FACE AUX NOUVEAUX FLUX MIGRATOIRES
}

\author{
MARRUECOS FRENTE A LOS NUEVOS FLUJOS MIGRATORIOS
}

MOROCCO: THE NEW MIGRATION FLOWS

\author{
Jamal Benamar \\ Abid Ihadiyan \\ Université Abdelmalek Essaâdi de Tánger. Marruecos/Morocco \\ byamal2000@yahoo.fr \\ ihadiyan_abid@yahoo.fr
}

Recibido/Received: 28/07/2016

Modificado/Modified: 10/09/2016

Aceptado/Accepted: 2/10/2016

\section{RÉSUMÉ}

La migration irrégulière ou illégale, alimentée ces dernières années par un flux massif de subsahariens qui partent du Nord de l'Afrique, y compris le Maroc, et à destination de l'Europe (Espagne et Italie comme points d'entrée privilégiés) commence à prendre des dimensions alarmantes et parfois dramatiques, compte tenu du prix à payer en vies pour traverser la Méditerranée.

Face à cette nouvelle réalité, le Maroc décide de réviser sa politique migratoire et d'engager des mesures législatives, institutionnelles et opératives pour donner une réponse à cette nouvelle situation pour le pays.

Nous analysons cette situation, à partir d'une enquête (ETF et AMERM, 2012), qui couvrit tout le territoire national, en nous nous centrons surtout sur la zone Nord du Maroc, s'intéressant aux migrants de retour et aux candidats à l'immigration irrégulière et de notre expérience dans un projet européen du programme POCTEFEX 2011-2013 auquel nous avons participé comme partenaire (Checa, 2014), on s'intéressera: au processus de control et de gestion des flux migratoires; aux principaux défis que suppose l'immigration en particulier subsaharienne pour le Maroc et aux défis et opportunités pour le Maroc devenu pays d'accueil des migrants.

\section{MOTS CLÉS}

Migrations irrégulières, nouvelle politique migratoire, défis, opportunités.

\section{SOMMAIRE}

1. Antécédents. 2. Eléments de méthodologie. 3. Tendance et politique migratoire. 4. Processus migratoire au Maroc. 5. Politique d'immigration et d'asile. 6. Constat. 7. Bibliographie.

\section{RESUMEN}

La migración irregular, alimentada estos últimos años por un flujo masivo de subsaharianos que parten del Norte de África, en la que se incluye Marruecos, y a destinación de Europa (España e Italia como 
puntos de entrada privilegiados) está tomando unas dimensiones alarmantes y a repetidas veces dramáticas, teniendo en cuanta el precio a pagar en vidas para cruzar el Mediterráneo.

Frente a esta nueva realidad, Marruecos plantea revisar su política migratoria y tomar medidas legislativas, institucionales e inclusive operativas para dar respuesta a esta nueva situación para el país.

Analizamos esta situación partiendo de una encuesta (ETF y AMERM, 2012), que abarcó todo el territorio nacional, aunque nos centraremos sobre todo en la zona Norte de Marruecos, que se interesó a los inmigrantes retornados, los candidatos a la inmigración irregular y de nuestra experiencia en un proyecto europeo del programa POCTEFEX 2011-2013 en el cual hemos participado como socio (Checa, 2014), se estudiará: la evolución del proceso de control y gestión de los flujos migratorios; los principales desafíos que supone la inmigración subsahariana para Marruecos y los desafíos y oportunidades para Marruecos como nuevo país de acogida de inmigrantes.

\title{
PALABRAS CLAVE
}

Migraciones irregulares, nueva política migratoria, desafíos, oportunidades.

\section{SUMARIO}

1. Antecedentes. 2. Elementos metodológicos. 3. Tendencia y política migratoria. 4. Proceso migratorio marroquí. 5. Política de inmigración y asilo. 6. Planteamiento final. 7. Bibliografía.

\begin{abstract}
Irregular migration, fueled in recent years by a massive influx of sub-Saharans who leave North Africa, where Morocco is included as destination in Europe (Spain and Italy as privileged points of entry) is taking alarming dimensions and repeatedly dramatic, taking into account the price paid in lives to cross the Mediterranean.

Faced with this new reality, Morocco raises revise its immigration policy and take legislative, institutional and even operational to respond to this new situation for the country measures.

We analyze this situation based on a survey (ETF et AMERM, 2012), covering the entire national territory, although we will focus especially in the north of Morocco, who was interested returnees immigrants, candidates for illegal immigration and our experience in a European project POCTEFEX 2011-2013 program to which you have participated as a partner (Czech, 2014), were studied: the evolution of process control and management of migration flows; the main challenges of sub-Saharan immigration to Morocco, and the challenges and opportunities for Morocco as new host country of immigrants.
\end{abstract}

\section{KEYWORDS}

Irregular migration, new immigration policy, challenges, opportunities.

\section{CONTENTS}

1. Background. 2. Methodological elements. 3. Trend and immigration policy. 4. Moroccan migration process. 5. Immigration and asylum policy. 6. Final Approach. 7. References.

\section{ANTÉCÉDENTS}

La question migratoire n'a pas cessé de faire couler beaucoup d'encre, au Maroc comme ailleurs. S'il est vrai que ce phénomène a pris des allures dramatiques avec ce que nous révèle les medias, avec son lot quotidien de migrants fuyant les guerres ou les migrants climatiques, ou encore et toujours les migrants économiques qui cherchent refuge en Europe, et risquent leurs vies dans la Méditerranée ou en traversant les Balkans, la question migratoire continue à diviser les pays européens incapables de lui trouver une solution durable. 
Le Maroc connaît aussi, et ce depuis longtemps, une dimension nouvelle du flux migratoire, caractérisée par un phénomène migratoire trans social et une présence accrue de migrants subsahariens. On estime entre 25000 et 40000 le nombre de migrants subsahariens en situation illégale au Maroc (La vie économique, 2014:4). Il est vrai que le flux migratoire marocain vers l'Europe s'est considérablement réduit, il n'en demeure pas moins que les immigrés de retour constituent une question importante qui a des impacts sur la société et les migrants eux-mêmes. Aussi, de nouveaux profils, candidats marocains à l'immigration illégale, se dessinent. D'autre part, la présence des subsahariens sur le territoire marocain, pour la plupart en situation de danger dans leurs pays, et eux- mêmes candidats au départ vers l'Europe à partir de sa frontière sud, oblige le Gouvernement marocain à revoir sa gestion de la question migratoire et en particulier sa politique d'immigration et d'asile, comme en témoigne la création en 2013, pour la première fois, d'un département en charge des Affaires de la Migration confié au Ministère des Marocains Résidants à l'Etranger.

Le Maroc deviendrait-il une nouvelle terre d'immigration? La réalité sur le terrain prouve que c'est vrai. Cette nouvelle donne place les autorités marocaines face au défi d'une bonne gouvernance de la question migratoire, d'autant plus que la société marocaine elle aussi est confrontée à un certain nombre de problématiques nouvelles. Il fallait donc changer de politique.

Comme illustration de cette réalité, l'ouvrage de (Bouba Oumarou, 2015), bien que romancé, illustre bien le parcours et les difficultés de la migration subsaharienne à Tanger et plus généralement au Maroc.

Nous avons voulu aborder dans cet article tous ces aspects. Notre analyse repose sur un travail d'envergure nationale (ETF et AMERM, 2012), Nous nous intéressons en particulier aux résultats issus des enquêtes sur le Nord du Maroc et de notre expérience de nos travaux précedents (Benamar, 2011; Ihadiyan, 2005-2009-2013), ainsi qu'une étude menée sur la question de l'intégration socio professionnelle des immigrés marocains en Andalousie (Checa y Olmos, 2014).

Face à cette nouvelle évolution du phénomène migratoire, il semblait intéressant de savoir ce qui a changé pour les candidats marocains à l'immigration irrégulière, comme par exemple leurs motivations, leurs destinations ainsi que leurs profils.

Les marocains de retour au pays, qui ont bénéficié des politiques des gouvernements européens d'aides au retour est perçu de manière mitigée. Les bénéfices attendus par ces pays n'étaient pas au rendez-vous, puisque d'une part, c'est une main d'œuvre pour la plupart relativement intégrée et bien formée (Benamar y Melero, 2015:79-92), puisque ayant séjourné des années dans le pays d'accueil, et que d'autre part, la migration irrégulière n'a pas cessé pour autant, apportant de nouveaux lots d'immigrés d'une " nouvelle génération », où la femme ne représente plus, comme par le passé (Hilal, 2007:181-204), une singularité dans les processus migratoires.

Ce constat, doublé d'une reprise prochaine des économies européennes, et donc de la nécessité de main d'œuvre pour assurer cette reprise (Benamar, 2012), a conduit ces gouvernements à revoir leur politique migratoire et à l'inscrire dans une démarche globale et concertée.

Ce travail présente les caractéristiques de la nouvelle vague d'immigration, de la migration de retour et ces impacts sur le pays, ainsi que la nouvelle approche du gouvernement marocain face à la gestion de la question migratoire. 


\section{ELEMENTS DE MÉTHODOLOGIE}

La méthodologie employée dans ce travail analyse une partie des résultats (Nord du Maroc) des travaux d'une étude à l'échelle nationale sur « la migration des compétences » (ETF et AMERM, 2012:60-76) auxquels a participé un des co auteurs de cet article. Nous avons également exploité des sources secondaires, de type statistique ou non en rapport avec nos objectifs, nos précédents travaux, les résultats des travaux d'un projet auquel nous avons participé (Checa y Olmos, 2014:251-265), ainsi qu'une bibliographie exhaustive sur le général et le particulier concernant le phénomène des migrations.

Concernant l'étude précitée qui a couvert huit régions du Maroc, elle est fondée sur une enquête statistique par sondage comportant deux volets: le premier auprès des migrants potentiels (échantillon fixé à 2600 personnes âgées de 18 à 50 ans) et le deuxième auprès des migrants de retour (échantillon de 1400 personnes). L'expression migrant de retour est employée pour toute personne de plus de 18 ans, ayant vécu et travaillé sans interruption au moins trois mois à l'étranger, revenue au Maroc durant la dernière décennie et présente au Maroc pour réaliser l'entretien.

\section{TENDANCE ET POLITIQUE MIGRATOIRE}

\subsection{Tendances migratoires}

La migration marocaine a connu globalement deux périodes de croissance :

a) Durant la période allant de 1968 à 1990, la migration marocaine a connu une croissance rapide, avec un taux de croissance annuel moyen de plus de $10 \%$.

b) Mais au cours des deux dernières décennies des migrations, c'est-à-dire de 1990 à 2011 cette migration a enregistré un accroissement moins rapide d'environ seulement $5 \%$ en moyenne par an.

Le nombre de marocains résidents à l'étranger est actuellement d'environ 3,5 millions d'individus dont $84 \%$ sont sur le territoire européen. Concernant la répartition par sexe, la migration marocaine est composée d'environ 58,6\% d'hommes et de $41,4 \%$ de femmes. S'agissant de son niveau d'instruction, seuls $18.5 \%$ sont analphabètes, les autres ont tous bénéficié d'une instruction scolaire. Si l'on tient compte de la migration clandestine, ce chiffre approcherait, en 2012 les 4,5 Millions de personnes, selon l'estimation du Ministère Chargé des Marocains Résidant à l'Etranger (MRE).

Mais ces chiffres concernent la migration régulière. Quant à la migration irrégulière, son caractère clandestin rend difficile la saisie de ses flux dans leur consistance quantitative et qualitative. Les approximations opérées à travers les régularisations effectuées par l'Espagne et l'Italie durant les années 90 et jusqu'à 2005 permettent d'atteindre un effectif de 297.165 (Mghari, 2009:2) migrants clandestins régularisés en 15 ans, soit un «stock » moyen annuel d'irréguliers en Europe de presque 20 milles migrants par an.

Des facteurs internes (sécheresse, chômage, carences des politiques sociales,...) alliés aux durcissements des conditions d'entrée en Europe, pour la migration européenne, poussent les migrants potentiels à opter généralement pour la voie de la clandestinité sous des formes et des itinéraires variés (Vallejo et Benamar, 2007:69-86).

Concomitamment avec ces développements, le flux des migrants subsahariens clandestins grandissait, au Maroc. L'objectif de ces migrants n'était pas de s'installer dans le pays mais de traverser le détroit pour atteindre l'Europe. Les estimations de ces flux en transit sont divergentes. Selon le Ministère de l'Intérieur, le nombre de ces migrants oscille entre 10.000 
à 15.000, selon l'Organisation Internationale pour les Migrations, ce chiffre oscille entre 10.000 et 20.000 .

Dans bien des cas, le transit se transforme en séjour forcé plus ou moins long. Les difficultés que pose la traversée du Détroit de Gibraltar ou de l'Océan Atlantique vers les Iles Canaries font que le Maroc et en particulier, les régions du nord et nord-est, les villes de Tanger, Nador, Casablanca, Rabat, Fès et Agadir deviennent une escale durable.

Depuis la crise économique et financière, quelques pays européens mirent en place des politiques d'aides au retour volontaire vers les pays d'origine, en même temps qu'ils durcissaient les conditions d'entrée et de séjour. L'efficacité de ces mesures n'a pas été encore établie, c'est pourquoi l'étude qui a été menée (ETF et AMERM, 2012) reste pionnière car elle analyse l'impact de la migration de retour et les profils des nouveaux candidats à l'émigration.

\subsection{Impératifs politiques et cadre législatif}

Face à des vagues humaines de plus en plus croissantes qui déferlent sur le continent européen et après plusieurs décennies de décisions unilatérales en matière de gestion des migrations, les pays européens ont pris conscience de la nécessité de gérer conjointement ce phénomène avec les pays d'origine des migrants afin d'en optimiser les impacts pour les deux côtés.

En effet, les pays européens, tout en reconnaissant le rôle de l'immigration pour combler le déficit démographique et répondre aux besoins de la croissance économique, voudraient en avoir une bonne maîtrise.

L'un des projets expérimentés dans ce cadre est celui de la migration temporaire organisée. Il s'agit d'un projet réalisé dans le cadre de la coopération entre l'UE et le Maroc via l'Agence Nationale de Promotion de l'Emploi et des Compétences (ANAPEC), visant à organiser le placement international selon les besoins des pays demandeurs. Le premier essai dans ce sens a porté sur la mobilisation d'ouvrières marocaines pour répondre aux besoins temporaires de l'agriculture espagnole.

Inaugurée en 2006, cette opération a connu une croissance soutenue jusqu'en 2008. Elle est en régression depuis. Le volume de placement a atteint, pour l'année 2011, un total de 6807 personnes avec une légère augmentation par rapport à l'année 2010. L'Espagne, accueillant plus de $82 \%$ de cet effectif, reste le premier pays de destination, loin devant les autres pays.

Le gouvernement marocain a, à son tour, affiché son intérêt pour la question migratoire dans le souci d'atteindre certains objectifs généraux. Cela s'est traduit par des décisions institutionnelle et juridique et une approche régionale voire internationale pour le traitement de ce phénomène. L'essentiel de ces objectifs sont : la régulation du marché du travail par la maitrise des flux migratoire, le maintien des liens humains et culturels des migrants avec leur patrie, développer et faciliter les transferts financiers et du savoir-faire et contrecarrer la migration irrégulière.

Pour ce faire, et d'un point de vue institutionnel dans le but de coordonner les actions des différents départements ministériels, le Gouvernent marocain a créé en 1990 un Ministère Chargé des Affaires de la Communauté Marocaine Résidant à l'Etranger.

D'autre part, et sous la pression de l'Union Européenne, l'année 2003 a été marquée par une stratégie de lutte contre l'émigration clandestine reposant sur la création, au sein du Ministère de l'Intérieur, de la Direction de la Migration et de la Surveillance des Frontières et l'adoption de la loi 02-03 de 2013 relative à l'entrée et au séjour des étrangers au Maroc, à l'émigration et l'immigration irrégulière. 
Cette loi, qui semble répondre, au moins en partie, à des pressions extérieures, s'inscrit dans une conjoncture internationale et régionale qui privilégie la dimension sécuritaire au détriment de celle des droits humains.

Certes le Maroc, en matière de migration, a pris des engagements aux niveaux bilatéral, régional et international. L'objectif principal de ces conventions est de protéger les travailleurs marocains à l'étranger et de fixer les modalités de leur recrutement, les conditions de leur travail ainsi que leur déplacement, leur établissement et les institutions qui se chargent d'eux.

D'autre part, au niveau régional et international, le Maroc a pris des engagements dans le cadre du conseil d'association avec l'Union Européenne sur "la gestion commune des flux migratoires" et a accepté de négocier "la réadmission des clandestins dans les pays d'origine ou de transit», ce qu'il s'était, jusqu'alors, toujours refusé à faire même s'il a signé un accord de réadmission avec l'Espagne en 1992 et avec l'Italie en 1998.

\section{PROCESSUS MIGRATOIRE AU MAROC}

Le flux migratoire marocain, en particulier à destination de l'Europe (González y Vera, 2008), a connu un fort ralentissement ces dernières années pour les raisons évoquées (crise économique, politiques européennes d'aide au retour, renforcement des frontières et durcissement dans l'attribution des visas, une meilleure collaboration entre les pays d'accueil et le Maroc dans la lutte contre l'immigration clandestine, ...). Aussi, le profil du candidat à l'immigration ainsi que les raisons le poussant à envisager un projet de départ vers l'Europe ont évolué avec le temps et les contextes politique, économique et social, aussi bien du pays d'origine que des pays d'accueil. Il apparaît donc naturel d'étudier ces aspects.

\subsection{Les nouvelles compétences à l'immigration}

Dans un échantillon où la proportion de femmes égale presque celle des hommes, les deux tiers sont d'origine rural et la moitié déclare être mariés, l'étude (ETF et AMERM, 2012:78) fait ressortir que 58\% des enquêtés n'ont pas envie de quitter le Maroc, alors que $42 \%$ (dont environ $32 \%$ avec un faible désir et $62 \%$ avec un fort potentiel d'émigration) ont manifesté l'intention de partir travailler à l'étranger. En définitive, sur l'ensemble de l'échantillon un peu plus de $13 \%$ ont émis une forte envie de réaliser un projet migratoire. La figure 1 reflète le potentiel d'émigration pour les 8 régions étudiées.

Figure $1:$ Immigrés potentiels par région

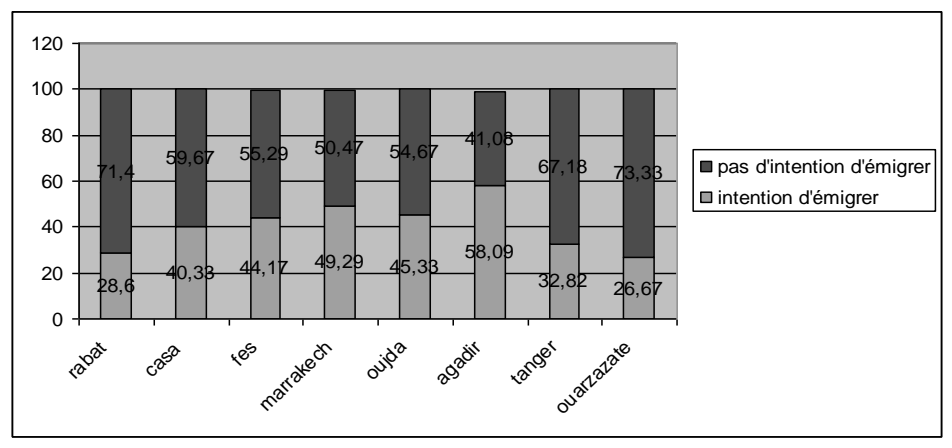

Source : ETF et AMERM, 2012, p 79. 
Nous avons porté une attention particulière aux caractéristiques de ces candidats à fort potentiel d'émigration pour comprendre « la personnalité » de ces nouveaux migrants de « nouvelle génération »:

a) Caractéristiques sociales

L'étude montre que les hommes ont une plus forte propension à partir à l'étranger chercher un nouvel avenir. Les deux tiers des personnes ayant envie d'émigrer sont des hommes. Sur les $40 \%$ de femmes qui ont envie d'émigrer, $39 \%$ ont un fort potentiel d'émigration.

La tendance à vouloir émigrer continue de pointer les jeunes (entre 18 et 29 ans) comme le collectif privilégié. Un peu plus de la moitié des jeunes ont l'intention d'émigrer.

Quant à la situation familiale, elle est en corrélation négative avec l'envie de partir à l'étranger, et ce indépendamment du sexe. Ce qui signifie que les personnes ayant une forte envie d'émigrer sont majoritairement des femmes et des hommes célibataires.

Les personnes de l'échantillon ont répondu à $40 \%$ que leur situation financière est parfois suffisante et parfois insuffisante. Les femmes à 33\% ayant un fort potentiel d'émigration pensent que la situation financière de leur foyer est insuffisante voir pas du tout suffisante.

b) Education

On constate parmi les personnes à fort potentiel d'émigration un taux significatif de personnes ayant un niveau d'éducation moyen à élever $(28 \%$ et $23 \%$; respectivement pour les femmes et les hommes).

Il apparaît que la grande majorité des enquêtés est conscient qu'un niveau élevé d'éducation au Maroc aide à trouver un bon emploi à l'étranger (79,37\%). Ce taux atteint $85 \%$ pour les candidats à l'émigration.

c) Aspects professionnels

D'après les résultats de l'étude, on constate que le projet migratoire ne semble pas être corrélé avec l'emploi des personnes enquêtées. En effet, le groupe des personnes avec un fort potentiel d'émigration est composé, à part égale, des personnes qui ont travaillé les 7 derniers jours et des personnes qui n'ont pas travaillé.

Pour les personnes actives, les secteurs d'activité sont variés, il s'agit essentiellement de l'agriculture, du commerce, du secteur manufacturier, du secteur des services et de la construction. L'enquête a révélé que les personnes ayant un projet migratoire travaillent dans ces secteurs. Les personnes ne souhaitant pas émigrer sont présentes surtout dans le secteur agricole et le secteur du commerce.

\subsection{Les migrants de retour}

L'étude a montré que la réussite de la migration de retour est le résultat de la combinaison de plusieurs facteurs:

- La durée de la migration: le retour est perçu comme négatif pour les migrants ayant séjourné de 1 à 3 ans (40\% de l'ensemble).

- Le niveau d'éducation: Le retour est plus réussi pour ceux qui ont un niveau d'éducation supérieur. Le taux de réussite est toutefois moins performant pour le retour que pour la migration. Ceci s'explique par les difficultés de réinsertion liées à un grand nombre de contraintes auxquelles se trouve confronté le migrant à son retour.

- Les nouvelles compétences acquises dans le pays d'accueil qui réduisent considérablement le temps nécessaire pour trouver un emploi. 
- Les programmes de soutien à la réinsertion: les institutions publiques marocaines ont développé des services d'accueil des migrants de retour en vue de leur réinsertion dans le milieu socio-économique national. L'étude a révélé une méconnaissance latente de ses services par les personnes enquêtées dont $6 \%$ seulement en connaissent l'existence.

- La transférabilité des droits sociaux (retraites, allocation chômage, ...): près de $80 \%$ de ceux ayant bénéficié de cette transférabilité n'ont pas l'intention de ré-émigrer.

Figure 2 : Niveau de formation des migrants de retour selon la tranche d'âge

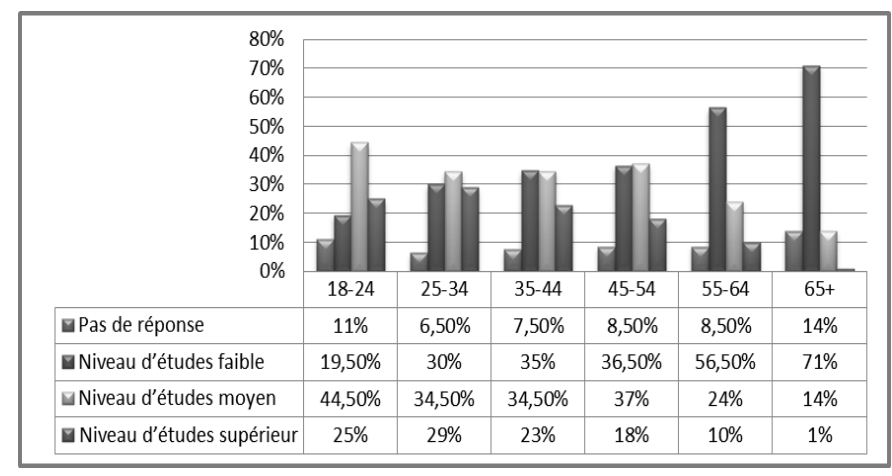

Source : ETF et AMERM, 2012, p 110.

\section{POLITIQUE D'IMMIGRATION ET D'ASILE}

L'évolution des flux migratoires au Maroc, depuis des décennies, et dans un contexte mondial, a incité le Gouvernement marocain à œuvrer pour gérer la question de la migration conformément à ses engagements nationaux (Constitution, nouvelle approche du phénomène, ...) et internationaux (Droits de l'Homme, Coopération, ...).

En effet, les flux migratoires se sont régionalisés, et le Maroc, de par sa position géographique au carrefour des pays du Sud et l'Europe, de stabilité politique et économique, passe d'être un pays de transit à un pays d'accueil.

Figure 3 : Evolution du nombre de réfugiés

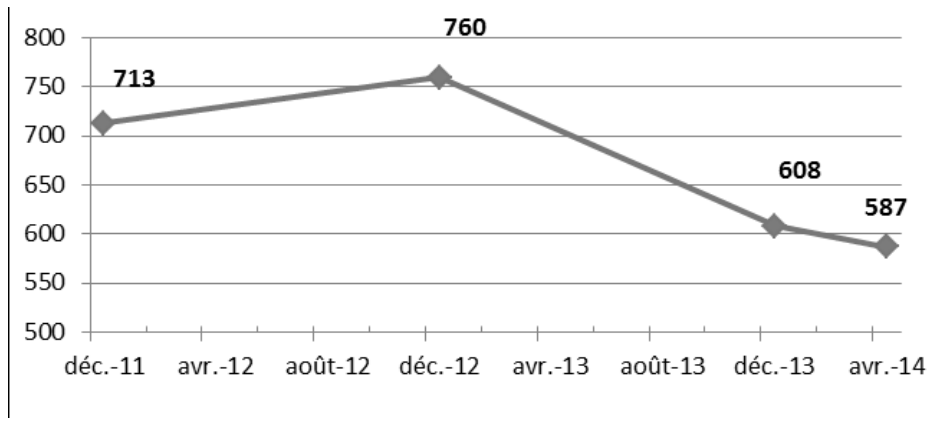

La migration africaine et tout récemment arabe, devait répondre aux défis de l'immigration dans le respect de la dignité humaine, de l'intégration sociale, économique et 
culturelle des migrants (Benamar, 2009:27-38). C'est dans ce contexte que le Gouvernement marocain, sous l'impulsion du Chef de l'État, a entrepris de mettre en place une stratégie de l'immigration et de l'asile qui doit donner des réponses intégrées et conformes aux droits humains.

C'est dans ce sens qu'il a été créé en septembre 2013 quatre sous-commissions chargées de la mise en place de la nouvelle politique nationale d'immigration et d'asile (NPIA), présentée à Genève le 17 mars 2014 (DEBBARH, 2014) : de la régularisation des étrangers en situation irrégulière, de la régularisation des réfugiés statutaires, reconnus par le HCR, de la mise à niveau du cadre juridique et institutionnel et sur l'action diplomatique.

Figure 4 : Nombre de demandeurs d'asile

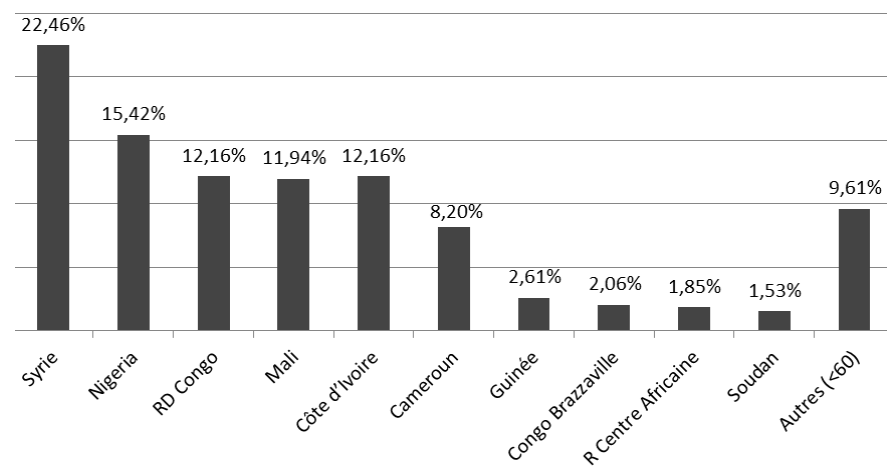

Sources figure 3 et 4 : DEBBARH J. (2014)

\subsection{La réponse du gouvernement}

L'action du Gouvernement dans le cadre de la NPIA repose sur quatre piliers :

- La régularisation des migrants et demandeurs d'asile: dès septembre 2013 il a été mis en place 83 bureaux des étrangers dans les préfectures du Royaume et que des moyens humains et logistiques ont été déployés. Deux opérations de régularisation ont été menées (régularisation des demandeurs d'asile reconnus par le Bureau du HCR et régularisation exceptionnelle des migrants irréguliers ; le nombre de personnes en situation irrégulière au Maroc est estimé entre 25.000 et 40.000).

- Le dispositif institutionnel et législatif: Création en 2013, pour la première fois, d'un département en charge des Affaires de la Migration confié au Ministère des Marocains Résidant à l'Etranger. Ce Ministère a pour mission d'assurer la coordination, la planification et la mise en œuvre de cette politique.

Et sur le plan juridique, un projet de Loi sur l'asile est préparé dans l'esprit de la Convention de Genève du 28 juillet 1951, par ailleurs ratifiée par le Maroc en 1958; un deuxième projet de Loi sur la traite des êtres humains et enfin un troisième projet de Loi sur l'immigration (Entrée et séjour au Maroc des étrangers) en remplacement de la Loi 02-03 loi 02-03 relative à l'entrée et au séjour des étrangers au Royaume du Maroc, à l'émigration et l'immigration irrégulière, car jugée peu respectueuse des droits humains.

- La politique d'intégration des migrants et des réfugiés: il s'agit d'intervenir dans l'éducation, la santé et logement, l'inter culturalité, la formation professionnelle, l'assistance sociale, juridique et administrative et la veille stratégique. 
Nous avons mené une expérience de ce type d'intégration socio professionnelle des immigrés marocains résidants en Andalousie (CHECA Y OLMOS, 2014 : 251-265); dans le cadre d'un projet européen POCTEFEX 2011-2013.

- Le partenariat et la coopération: mise en place de partenariats institutionnels, de partenariat public-privé, de partenariat avec les Organisations de la Société Civile et de partenariat international (OIM, NU, EU).

\subsection{Le Maroc face aux nouveaux flux migratoires}

Le Maroc, c'est un fait, est entré dans le « club » des pays récepteurs d'une immigration variée en quête d'un meilleur projet de vie ou fuyant la situation de danger de vie sévissant dans leurs pays d'origine. Il n'avait pas vocation à cela, mais sa coopération avec les européens pour une meilleure surveillance de ses frontières, les mesures de plus en plus restrictives de délivrances des visas ou encore le renforcement de la surveillance des frontières de l'Europe, ont obligé les migrants "à allonger » leur séjour dans l'attente de trouver l'opportunité pour atteindre l'Europe. Ce qui devait être pour le migrant juste un transit par le Maroc est devenu une résidence forcée.

Cela ne va pas sans créer pour la société marocaine un ensemble totalement nouveau de problématiques sociales et juridiques qui sont typiques des pays d'immigration et qui ne correspondent pas encore à l'image que le Maroc nourrit de lui-même, qui est celle d'un pays d'immigration. Conscient de cette transformation en terre d'accueil, le Maroc a choisi de traiter ce phénomène avec clairvoyance, responsabilité et respect des Droits Humains, en adoptant, sous l'impulsion du Roi du Maroc, la nouvelle politique migratoire.

Mais à ces flux migratoires légaux ou illégaux de nature économique, se sont greffés de manière exponentielle ces dernières années des flux de réfugiés climatiques, de guerre ou de catastrophe naturelle. Le Maroc doit faire face à ces nouveaux défis humanitaires et donner des réponses politiques, économiques et sociales à ces nouveaux phénomènes. Nous pensons intégrer l'étude de ce phénomène dans nos prochains travaux de recherche.

\section{CONSTAT}

Compte tenu des incohérences des politiques migratoires et le constat mitigé des résultats de ces politiques mises en œuvre jusqu'à nos jours, il s'avère impératif de repenser ce cadre légal et réglementaire afin de pallier ses insuffisances et élaborer un cadre réglementaire des flux migratoires entre le Maroc et ses partenaires européens. Plusieurs facteurs (ETF et AMERM, 2012:169-171), militent en faveur de cette institutionnalisation de la mobilité avec les droits que cela engendre pour le migrant :

- Les déficiences dues à une politique focalisée davantage sur des mesures restrictives dont les effets sont parfois contraires aux intentions de départ, engendrant le développement de l'émigration illégale.

- Cette politique a un coût élevé pour les contribuables européens. Des moyens de contrôle et de régulation toujours plus sophistiqués et donc coûteux sont mis en place mais dont l'efficacité est limitée par la détermination des migrants à réaliser leurs projets de migration, par la multiplicité des formes et des voies d'entrée en Europe.

- Elle a aussi un coût très élevé en vies humaines, car cette aventure se termine assez souvent de manière tragique au fond des eaux du Détroit ou de l'Océan au large des Iles Canaries ou des îles italiennes (Khachani, 2006). 
- Le défi démographique. La croissance de la population demeure importante au sud de la Méditerranée: elle a été en deux décennies de 1980 à 1999, de 45\% au Maroc, elle est estimée à plus de $35 \%$ entre 2000 et 2025 . Cette croissance sera soutenue en dépit de l'état d'avancement de la transition démographique. Mais il convient de rappeler que l'aubaine démographique au Maroc devrait durer jusqu'en 2017, une "aubaine", pour qui il faut créer des emplois (près de 400.000 par an). En Europe, la croissance démographique en l'espace de 25 ans serait à peine de 3,5\% faisant passer la population de 375 millions en 2000 à 388,3 millions d'habitants en 2025.

- Une demande de travail spécifique existe dans les pays de l'UE, répondant aux besoins d'un marché d'emploi secondaire, où prédominent des emplois essentiellement précaires et/ou socialement indésirables. Mais où de plus en plus, ces recrutements intéressent également des compétences de différents niveaux.

- Du point de vue géostratégique, le référentiel dans la région demeure la déclaration de Barcelone dont l'objectif fondamental est de faire de l'espace euro-méditerranéen un espace de paix et de prospérité partagée.

Force est de constater que le libre-échange ne réduira pas les pressions migratoires sur le court terme, mais il les accroîtra vraisemblablement. Dans un premier temps, il entraînera une perte de compétitivité des industries du Sud accompagnée d'une diminution de l'emploi qu'elles assurent, malgré les programmes de « mise à niveau » des entreprises. Cet état de choses augmentera la pression migratoire vers l'Europe (Ihadiyan, 2001).

Les pays d'origine des migrants, de même que les pays de destination, ont donc tout intérêt à œuvrer de concert pour veiller à ce que " les transferts de fonds, de savoir-faire et d'innovations procurent un maximum d'avantages en termes de développement ». Cela semble la méthode par excellence pour réduire les processus migratoires (Philip et Strabhaar, 2002:5-23).

\section{BIBLIOGRAPHIE}

BENAMAR, J. y MELERO AGUILAR, N. (2015). "El fenómeno migratorio entre Andalucía y Marruecos: reflexiones para un desarrollo humano entre ambas orillas". Barataria. Revista CastellanoManchega de Ciencias Sociales, 20: 79-92, DOI: http://dx.doi.org/10.20932/barataria.v0i20.14

BENAMAR, J. (2012). "Contratación de inmigrantes marroquíes: Oportunidades para las PYMEs andaluzas". Revista Cámara Sevilla, Sevilla, Diciembre 2012, vol. 45:22-23.

BENAMAR, J. (2011). "Inmigración e integración en el futuro del Mediterráneo". Estudios sobre inmigración. Selección de textos de los Simposios Internacionales 2007-2010. Madrid: Fundación Ciudadanía y Valores, pp. 95-121.

BENAMAR, J. (2009). "Inmigración, integración e interacción en el futuro del Mediterráneo". Barataria. Revista Castellano-Manchega de Ciencias Sociales, 10: 27-38, DOI: http://dx.doi.org/10.20932/barataria.v0i10.165

BOUBA OUMAROU, S. (2015). "Boukhalef jusqu'au bout". Le Journal de Tanger. Tánger.

CHECA Y OLMOS, F. (2014). Los inmigrados marroquíes en Andalucía. Una investigación necesaria, Almería. Almería: Universidad de Almería.

DEBBARH, J. (2014). "La nouvelle politique d'Immigration et d'Asile du Royaume du Maroc, Politique de gestion de la question migratoire", in http://www.processusderabat.net/web/uploads/SOMRabat/La-nouvelle-politique-d-immigration-et-d-asile-du-Maroc-(partie-1).pdf (consulté 26/03/2015). ETF et AMERM (2012). Migrations et compétences au Maroc. Rabat: Edition European Training Foundation. Rapport National.

GONZÁLEZ, G. y VERA, M. (2008). África, 14 kilómetros al Sur de Europa. Análisis de los flujos migratorios entre el Norte de África y el Sur de Andalucía, Sevilla: Junta de Andalucía. 
HILAL, L. y BENAMAR, J. (2007). "Stratégies d'actions pour une reconstruction de l'identité de genre de la femme rurale marocain", en L. Hilal y F.A. Vallejo Pena (Coords.) L'identité de genre de la femme marocaine actuelle. Tanger: Editeur Ifzarne, pp.181-204.

IHADIYAN, A. (2001). Libre-échange et migration : une simulation pour le Maroc. Thèse de doctorat, Dunkerque: Université du Littoral Côte d'Opale.

IHADIYAN, A. (2005). "Émigration et développement économique : enjeux pour la région maghrébine". Revue Critique économique, vol.16. Rabat.

IHADIYAN, A. (2009). "Transferts de revenus des migrants et développement local : résultats d'une enquête sur la région de Nador". Publication de l'AMERM, Rabat

IHADIYAN, A. (2013). Libre échange et migration. Saarbrücken: Editions universitaires européennes.

KHACHANI, M. (2006). "La migración subsahariana: Marruecos como espacio de tránsito". Publicaciones CIDOB. Barcelona.

LA VIE ECONOMIQUE (2014). "Le Maroc nouvelle terre d'Immigration”. La vie économique du 09 août, in http://www.lavieeco.com/news/societe/le-maroc-nouvelle-terre-d-immigration-30643.html (consulté 11/05/2015).

LE MONDE (2014). "Le Maroc met en œuvre une nouvelle politique d'accueil des subsahariens", in https://goo.gl/xcdK3c (consulté 11/05/2015).

MARMOLEJO MARTÍN, J.A. (2009). "Patrón de entradas en la inmigración ilegal de la ciudad autónoma de Melilla", en J. J. Fernández Alles (Coord.) El Estrecho de Gibraltar como espacio jurídico común. Valencia: Tirant Lo Blanch, pp. 363-391.

MGHARI, M. (2009). "Migration Irrégulière Marocaine". Publication CERED, Rabat, in http://iussp2009.princeton.edu/papers/92040 (consulté mai 2015).

PHILIP, M. et STRABHAAR, T. (2002). "Best practices to reduce migration pressures". International Migration, Vol. 40 (3): 5-23.

VALLEJO PEÑA, F. A. y BENAMAR, J. (2007). "Los flujos migratorios ilegales en el Estrecho de Gibraltar". Barataria. Revista Castellano-Manchega de Ciencias Sociales, 8: 69-86, DOI: http://dx.doi.org/10.20932/barataria.v0i8.213

\section{Breve currículo:}

\section{Jamal Benamar}

Catedrático de Informática de gestión. Profesor de Comunicación Interpersonal, Jefe del Departamento de Economía y Gestión y Director del grupo de Investigación en Economía y Territorio.

Coordinador mediterráneo y ponente de varios cursos de postgrado entre entes públicos españoles, marroquíes e internacionales y de seminarios sobre inmigración, mediación, gestión y administración pública, PCI España-Marruecos. Autor de múltiples artículos sobre temas de inmigración, género y desarrollo sostenible en revistas de ámbito nacional e internacional.

\footnotetext{
Abid Ihadiyan

Profesor de Ciencias Económicas y de Gestión. Coordinador del grupo de Investigación sobre la Integración regional, Empleo, Inmigración y Desarrollo. Autor de múltiples libros y artículos sobre temas de inmigración, desarrollo y comercio internacional en varias revistas de ámbito nacional e internacional.
} 